\title{
Wideband DOA estimation algorithm based on signal subspace rebuilding
}

\author{
Zhi-Qiang Liu ${ }^{1}$, Hong-Guang $\mathrm{Ma}^{2}$ and Jian Yang ${ }^{1}$ \\ ${ }^{I} X i^{\prime}$ an Research Institute of High Technology, Xi'an, 710025, PR China \\ ${ }^{2}$ Beijing Institute of Technology, Zhuhai, 519088, China \\ E-mail: Liuzhiqiang_xgx@163.com
}

\begin{abstract}
A new direction-of-arrival (DOA) estimation algorithm based on signal subspace rebuilding (SSR) is proposed for wideband sources. In the proposed algorithm, the signal subspaces are filtered by SSR to reduce the effect of the direction vectors of nonsupposed directions. The signal subspaces of each frequency bin are focused and averaged to enhance the Signal-to-Noise Ratio (SNR) of signal subspace. Therefore the quality of spatial spectrum is enhanced. The algorithm does not require initial estimation of the DOAs. The performance of some wideband algorithms is compared with that of the algorithm through computer simulations. The simulation results show that the proposed algorithm has a higher resolution with lower bias and variance than other wideband algorithms.

Keywords: Wideband; Direction-of-arrival (DOA) Estimation; Signal Subspace Rebuilding.
\end{abstract}

\section{Introduction}

Direction-of-arrival (DOA) estimation plays a very important role in both electronic warfare (EW) systems and mobile communication systems. In order to estimate DOAs, many narrow band methods are proposed[1-4]. The narrow band prerequisite is not suitable for the fact that wideband signals have been widely used nowadays in many fields such as sonar, radar, and wireless communications [5]. In order to estimate DOAs of wideband sources, many algorithms were proposed, such as incoherent signal subspace methods (ISSM) [6], coherent signal subspace methods(CSSM) [7-10], beamforming method[11], orthogonality testing method [12,13], jamming direction finding (JDF) and simplified JDF (SJDF) [14]. The efficiency of ISSM deteriorates for closely separated sources and low signal-to-noise ratio (SNR) [8]. CSSMs require initial values to build focusing matrices, and the estimation performance is sensitive to 
the initial values [12]. Beamforming method MVDR [11] is based on Capon beamforming, so it is difficult to get high resolution. TOPS cannot estimate the directions when the number of sources is greater than the half of array number [12], and it has many false peaks [13]. The disadvantage of TOFS is that it cannot get a clear peak when one or more narrowband spectra miss peaks for the rank of narrow spectrum vector will be one here. JDF and SJDF cannot work when there are multi-signals (not one signal) in same narrowband [14]. In this paper, a new wideband DOA estimation algorithm is proposed, which is mainly based on signal subspace rebuilding (SSR). The advantages of the new algorithm are 1) it doesn't need initial DOA values, like JDF, TOFS and TOPS, 2) it can achieve a high resolution, lower bias and variance than other wideband DOA estimators mentioned above.

\section{Wideband Signal Model}

Consider an array of $p$ sensors exposed to $q$ far-field independent wideband sources. The array output of jth narrow band in frequency domain is represented by

$$
\mathbf{x}\left(\omega_{j}\right)=\mathbf{A}\left(\omega_{j}, \boldsymbol{\theta}\right) \mathbf{s}\left(\omega_{j}\right)+\mathbf{n}\left(\omega_{j}\right)
$$

Where, $\mathbf{x}\left(\omega_{j}\right)$ is the received frequency signal, $\mathbf{A}\left(\omega_{j}, \boldsymbol{\theta}\right)$ is array manifold matrix, $\mathbf{s}\left(\omega_{j}\right)$ is the sources' signal, and $\mathbf{n}\left(\omega_{j}\right)$ is noise.

The correlation matrix of jth frequency signal (1) is

$$
\begin{aligned}
\mathbf{R}_{j} & =E\left[\mathbf{x}\left(\omega_{j}\right) \mathbf{x}^{H}\left(\omega_{j}\right)\right] \\
& =\mathbf{A}\left(\omega_{j}, \boldsymbol{\theta}\right) E\left[\mathbf{s}\left(\omega_{j}\right) \mathbf{s}^{H}\left(\omega_{j}\right)\right] \mathbf{A}^{H}\left(\omega_{j}, \boldsymbol{\theta}\right)+\sigma^{2} \mathbf{I} \\
& =\mathbf{A}\left(\omega_{j}, \boldsymbol{\theta}\right) \mathbf{R}_{\mathbf{S S}}\left(\omega_{j}\right) \mathbf{A}^{H}\left(\omega_{j}, \boldsymbol{\theta}\right)+\sigma^{2} \mathbf{I}
\end{aligned}
$$

Where, the superscript $H$ denotes conjugate transpose, $\sigma^{2}$ is the power of noise and I denotes identity matrix.

If the sources of (1) are uncorrelated, $q \times q$ matrix $\mathbf{R}_{\mathrm{ss}}$ must be full rank, and $\mathbf{R}_{j}$ in (2) can be decomposed by eigenvalue decomposition (EVD)

$$
\mathbf{R}_{j}=\left[\mathbf{U} \mathbf{s}_{j} \mid \mathbf{U} \mathbf{n}_{j}\right]^{H} \boldsymbol{\Sigma}\left[\mathbf{U} \mathbf{s}_{j} \mid \mathbf{U} \mathbf{n}_{j}\right]
$$

Where $\mathbf{U} \mathbf{s}_{j}$ is $p \times q$ matrix called signal subspace, $\mathbf{U} \mathbf{n}_{j}$ is $p \times(p-q)$ matrix called noise subspace and $\boldsymbol{\Sigma}=\operatorname{diag}\left(\lambda_{j, 1}+\sigma^{2}, \lambda_{j, 2}+\sigma^{2}, \ldots, \lambda_{j} q+\sigma^{2}, \sigma^{2}, \ldots, \sigma^{2}\right)$

\section{Signal Subspace Rebuilding Algorithm}

The outline of SSR is reducing the deviation of signal subspaces by averaging each signal subspace to get more accurate spatial spectrum. From signal models 
(1) and (3), we can see that signal subspace $\mathbf{U} \mathbf{s}_{j}$ spans a space as same as $\mathbf{A}\left(\omega_{j}, \boldsymbol{\theta}\right)$ spans, in a noise-free environment. However, in real sample signal, the estimated $\mathbf{U} \mathbf{s}_{j}$ that spans a space isn't equal to $\mathbf{A}\left(\omega_{j}, \boldsymbol{\theta}\right)$ spans. We can focus the signal space on a same frequency and average the estimated signal subspaces to get more accurate signal subspace.

However, it is difficult to get exact focusing matrix without initial DOAs. In one source occasion, JDF and SJDF select the focusing matrix by scanning. In multi-source occasion, we assume that there is only one signal whose DOA is $\theta$ in the space, and then rebuild the signal subspace which is made up of direction vector $\mathbf{a}\left(\omega_{j}, \theta\right)$ and noise. After rebuilding, the signal subspace is not affected by other directions' signal. The signal subspace is rebuilt as follows

$$
\mathbf{u}_{j}(\theta)=\mathbf{U} \mathbf{s}_{j} \mathbf{U} \mathbf{s}_{j}^{H} \mathbf{a}\left(\omega_{j}, \theta\right)
$$

After rebuilding, signal subspace $\mathbf{u}_{j}(\theta)$ and $\mathbf{a}\left(\omega_{j}, \theta\right)$ are in same space, in a noise-free environment.

The signal subspace is focused as

$$
\mathbf{U}_{j}(\theta)=\operatorname{diag}\left(\mathbf{a}\left(-\omega_{j}, \theta\right)\right) \mathbf{u}_{j}(\theta)
$$

It is easy to proof $\left|\mathbf{U}_{j}(\theta)\right|^{2}=p$, in a noise-free occasion. There is a rotate factor $c_{j}$ satisfy $c_{j} \mathbf{U}_{j}(\theta)=\mathbf{b}=\left[\begin{array}{llll}1 & 1 & \ldots & \mathbf{1}\end{array}\right]^{T}$, and its modulus must be 1 .

In real sample signal occasion, $\mathbf{U}_{j}(\theta)$ must be affected by noise. Therefore, $c_{j} \mathbf{U}_{j}(\theta)$ is made up of $\mathbf{b}$ and noise in real occasion. We can apply least square method to solve $c_{j}$

$$
\min _{c_{j}}\left\|\mathbf{b}-c_{j} \mathbf{U}_{j}(\theta)\right\|_{2} \text { subject to } \quad\left|c_{j}\right|=1
$$

The solution to (6) is given by

$$
c_{j}=\frac{\mathbf{U}_{j}^{H}(\theta) \mathbf{b}}{\left|\mathbf{U}_{j}^{H}(\theta) \mathbf{b}\right|}
$$

We can average the focused subspace to reduce the effect of noise. The signal subspace is averaged as follow:

$$
\mathbf{u}_{\mathbf{m}}(\theta)=\sum_{j=1}^{J} c_{j} \mathbf{U}_{j}(\theta)=\sum_{j=1}^{J} c_{j} \operatorname{diag}\left(\mathbf{a}\left(-\omega_{j}, \theta\right)\right) \mathbf{u}_{j}(\theta)
$$

After the processing of (8), the SNR of signal subspace $\mathbf{u}_{\mathbf{m}}(\theta)$ is enhanced, but its modulus cannot be $1 . \mathbf{u}_{\mathbf{m}}(\theta)$ is normalized to get the final signal subspace $\mathbf{u}_{\text {SSR }}(\theta)$.

$$
\mathbf{u}_{\mathrm{SSR}}(\theta)=\mathbf{u}_{\mathbf{m}}(\theta)\left|\mathbf{u}_{\mathbf{m}}(\theta)\right|^{-1}
$$


We can use the final signal subspace $\mathbf{u}_{\mathbf{S S R}}(\theta)$ to calculate the spatial spectrum:

$$
P_{\mathrm{SSR}}(\theta)=-10 \log \left(p-\left|\mathbf{b}^{T} \mathbf{u}_{\mathrm{SSR}}(\theta)\right|^{2}\right)
$$

Because we don't know the real DOAs in the space beforehand, we should calculate the spectrum for each $\theta$, and search the maximum of $P_{\mathrm{SSR}}(\theta)$ to get DOAs.

\section{Simulation}

It is assumed the array is ten-sensor uniform linear array (ULA) with inter space $0.15 \mathrm{~m}$. There are 4 far-field signals which directions are $-43^{\circ},-28^{\circ},-22^{\circ}$ and $31^{\circ}$, and powers are 1, 3,2 and 1, respectively. The signal spectrum is in the range of $600 \mathrm{MHz}$ to $800 \mathrm{MHz}$. The signal is converted to zero intermediate frequency signal with I and Q channels. The baseband signals of I and Q channels are sampled by Nyquist sampling frequency $200 \mathrm{MHz}$. The output of each sensor is separated into 256 snapshots of 32 samples. Then, an FFT algorithm is applied in each snapshot to sample the spectrum of the observation. The statistical performance was evaluated by performing 200 Monte Carlo runs for each scenario. The success times are counted when each estimated direction error is less than $2.5^{\circ}$, and all of the DOAs can be identified by the strongest peaks for the trials. The probability of resolution is defined as the ratio of success times to 200. The estimated deviation was calculated only based on the success results. For TOFS is better than ISSM and CSSM[13], we compare SSR with three new algorithms, MVDR, TOPS and TOFS. To compare easily, we use logarithmic spatial spectrum for other algorithms. The spatial spectrum of MVDR is defined as:

$$
P_{\mathrm{MVDR}}(\theta)=10 \log \sum_{j=1}^{J} \frac{1}{\mathbf{a}^{H}\left(\omega_{j}, \theta\right) \mathbf{R}_{j}^{-1} \mathbf{a}\left(\omega_{j}, \theta\right)}
$$

The spatial spectrum of TOPS is defined as:

$$
P_{\text {TOPS }}(\theta)=-10 \log \left(v_{\text {TOPS }}(\theta)\right)
$$

Where, $v_{\text {TOPS }}(\theta)$ is the spatial spectrum which is defined as the smallest singular value of $\mathbf{D}(\varphi)$ TOPS[12].

The spatial spectrum of TOFS is defined as:

$$
P_{\mathrm{TOFS}}(\theta)=-10 \log \left(v_{\mathrm{TOFS}}(\theta)\right)
$$

Where, $v_{\mathrm{TOFS}}(\theta)$ is the spatial spectrum which is defined as the smallest singular value of $D(\theta)$ in [13]. 
The simulation results are shown in Fig.1. To make the article brief, here only shows the biases and standard deviations of $-22^{\circ}$.

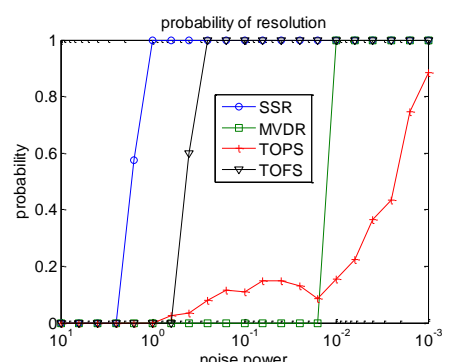

(a) the success probability for each algorithm

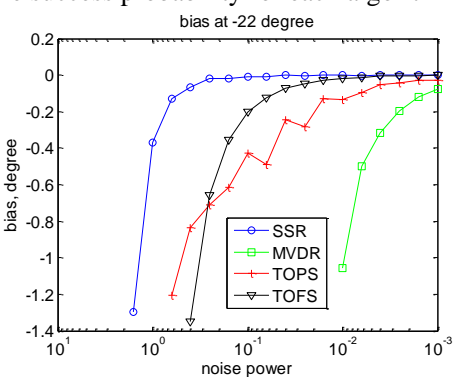

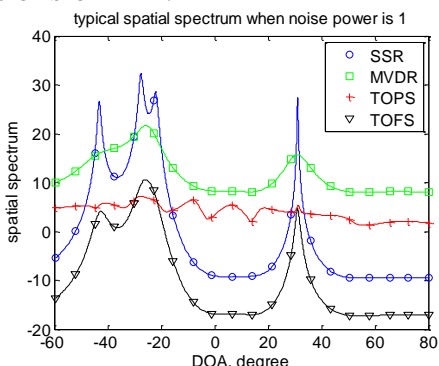

(b) typical spatial spectra for each algorithm $\left(\sigma^{2}=1\right)$

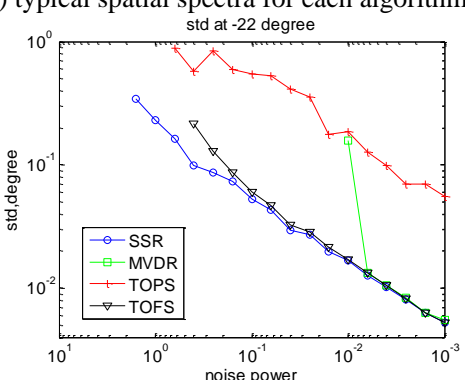

(c) biases for each algorithm at $-22^{\circ}$ (d) standard deviations (std) for each algorithm at $-22^{\circ}$ Fig. 1 Simulation Results

From the simulation results, we can see that SSR has lower resolution threshold, lower bias and lower standard deviation than other wideband processing methods. It gets higher performance than other wideband spectrum estimators. The reason is that SSR rebuilds and focuses the signal subspace, and averages the focused subspaces to get high SNR signal subspace. SSR can get better spatial spectrum than other algorithms.

\section{Conclusion}

We propose a new wideband DOA estimation algorithm, SSR. Unlike other wideband methods, this algorithm rebuilds and averages one-source signal subspace to enhance SNR of the signal subspace. Therefore it can get high quality spatial spectrum, and estimate DOAs more accurately than other wideband methods. The advantages of the new algorithm are summarized as follows: 1) it doesn't need initial DOA values, like JDF, TOFS and TOPS, 2) it 
can achieve a higher resolution with lower bias and variance than other wideband DOA algorithms.

\section{Acknowledgments}

We gratefully acknowledge the support of the National Natural Science Foundation of China (No.11405267 and No.61501471).

\section{References}

1. Canpon J.: 'High-resolution frequency-wavenumber spectrum analysis', Proceedings of IEEE, 1969, 57, (8), pp: 1408-1418, doi: 10.1109/PROC.1969.7278.

2. Schmidt R. O.: 'Multiple emitter location and signal parameter estimation', IEEE Trans. Antennas Propagat., 1986, 34, (3), pp. 276-280, doi: 10.1109/TAP.1986.1143830.

3. Gonen E, Mendel J M.: 'Applications of cumulants to array processing. VI. polarization and direction of arrival estimation with minimally constrained arrays'. IEEE. Trans. on Signal Process., 1999, 47, (9), pp: 2589-2592, doi: 10.1109/78.622949.

4. Liu C., Ye Z.F., Zhang Y.F.: 'DOA estimation based on fourth-order cumulants with unknown mutual coupling', Signal Processing., 2009, 89, pp: 1839-1843, doi: 10.1016/j.sigpro.2009.03.035.

5. Krim H., Viberg M.: 'Two decades of array signal processing research: The parametric approach', IEEE Trans. Signal Process., 1996, 13, (4), pp: 67-94, doi: 10.1109/79.526899.

6. Wax M., T. Shan, Kailath T.: 'Spatio-temporal spectral analysis by eigenstructure methods', IEEE Trans. Acoust., Speech. and Signal Process., 1984, 32, pp. 817-827. doi: 10.1109/TASSP.1984.1164400.

7. Wang H., Kaveh M.: 'Coherent signal-subspace processing for the detection and estimation of angles of arrival of multiple wideband sources', IEEE Trans. Acoust., Speech, Signal Process., 1985, 33, (4), pp.823-831. doi: 10.1109/TASSP.1985.1164667.

8. Shahrokh Valaee, Peter Kabal, "Wideband Array Processing Using a Twosided Correlation Transformation," IEEE Transactions on Signal Process., 1995, 43, (1), pp:160-172, doi: 10.1109/78.365295. 
9. Lee T. S.: 'Efficient wideband source localization using beamforming invariance technique', IEEE Trans. Signal Process., 1994, 42, (6), pp. 1376 1387, doi: 10.1109/78.286954.

10. Ng, W., Reilly, J.P., Kirubarajan, T., 'Wideband Array Signal Processing Using MCMC Methods', IEEE Trans. Signal Process., 2005, 53, (2), pp: 411-426, doi: 10.1109/TSP.2004.838934.

11. Chen H.W., Zhao J.W.: 'Wideband MVDR beamforming for acoustic vector sensor linear array', IEE Proc. Radar Sonar Navig., 2004, 151, (3), pp:158-162, doi: 10.1049/ip-rsn:20040651.

12. Yoon Y-S, Kaplan L. M., McClellan J. H., 'TOPS: new DOA estimator for wideband signals', IEEE Trans. Signal Process., 2006, 54, (6), pp: 19771989, doi: 10.1109/TSP.2006.872581.

13. Yu H.Q., Huang Z.T.: 'TOFS: a New DOA Estimator for Wideband Sources', Journal of Astronautic, 2007, 28, (5), pp: 1304-1308.

14. Liu Z.Q. Gao X.G., Ma H.G., 'JDF And SJDF: Two DOA Estimators for Wideband Jamming'. 10th International Conference on Signal Processing, Beijing, China, October, 2010 pp. 327-331, doi: 10.1109/ICOSP.2010.5657160. 